\title{
High spectral resolution ozone absorption cross-sections - Part 2: Temperature dependence
}

\author{
A. Serdyuchenko, V. Gorshelev, M. Weber, W. Chehade, and J. P. Burrows \\ Institute of Environmental Physics, Bremen University, Germany \\ Correspondence to: A. Serdyuchenko (anserd@iup.physik.uni-bremen.de) \\ Received: 12 May 2013 - Published in Atmos. Meas. Tech. Discuss.: 19 July 2013 \\ Revised: 8 November 2013 - Accepted: 26 November 2013 - Published: 24 February 2014
}

\begin{abstract}
We report on the temperature dependence of ozone absorption cross-sections measured in our laboratory in the broad spectral range $213-1100 \mathrm{~nm}$ with a spectral resolution of $0.02-0.24 \mathrm{~nm}$ (full width at half maximum, FWHM) in the atmospherically relevant temperature range from $193 \mathrm{~K}$ to $293 \mathrm{~K}$. The temperature dependence of ozone absorption cross-sections was established using measurements at eleven temperatures. This investigation is superior in terms of spectral range and number of considered temperatures compared to the previous studies. The methodology of the absolute broadband measurements, experimental procedures and spectra processing were described in our companion paper together with the associated uncertainty budget. In this paper, we report in detail on our data below room temperature and compare them with literature data using direct comparisons as well as the standard approach using a quadratic polynomial in temperature fitted to the cross-section data.
\end{abstract}

\section{Introduction}

Accurate information on the temperature dependence of the absorption cross-sections is vital for the retrievals of ozone profiles and columns from ultraviolet (UV) and visible (vis) spectra measured by modern remote sensing instruments. The origin of the temperature dependence of the ozone absorption cross-section arises from changes in the population distribution in the rotational vibrational states of the ground electronic state. The features and structure of the ozone absorption spectra and their changes with temperature were subject of extensive studies, mainly stimulated by ozone remote sensing applications (Barnes and Mauersberger, 1987; Brion et al., 1993; Bogumil et al., 2003, Burkholder and
Talukdar, 1994; Burrows et al., 1999; Malicet et al., 1989, 1995; Paur and Bass, 1984; Voigt et al., 2001). The Hartley absorption band in the UV comprises a very broad continuum with series of narrow peaks superimposed near the absorption maximum. At the maximum of the Hartley band around $254 \mathrm{~nm}$, the temperature variation is in the range of $0.5-1 \%$ from 203 to $273 \mathrm{~K}$ (Barnes and Mauersberger, 1987; Malicet et al., 1989). The temperature variation in the Huggins band is strongly wavelength-dependent with differences up to $70 \%$ and $30 \%$ for minimum and maximum of the spectral feature around $335 \mathrm{~nm}$ (Brion et al., 1993; Malicet et al., 1995; Paur and Bass, 1984). The Chappuis absorption band in the visible consists of a continuum with a series of peaks superimposed on the blue side of the spectrum. Weak temperature dependence below $1 \%$ was found for absorption cross-sections on the top of the band (Burkholder and Talukdar, 1994). The continuum of the visible is extended in the near-infrared (NIR) wavelength region, and on this extension the structure is superimposed. The Wulf absorption band in NIR exhibits temperature dependence similar to the Huggins band.

Table 1 provides an overview of the most relevant ozone cross-sections in the UV, visible, and near IR available at more than one temperature. More details on these datasets can be found in the review by Orphal (2003). In our companion paper (Gorshelev et al., 2014), we found typical accuracies of $2-3 \%$ in the spectral regions with strong absorption (maximum of Hartley, Huggins, and Chappuis bands) for all literature data, including our own measurements.

Among the datasets available at high spectral resolution (below $0.1 \mathrm{~nm}$ ), only the data from Brion, Malicet, and Daumont team (often abbreviated as BMD) were obtained from absolute measurements. Unfortunately, these data are 
Table 1. Characteristics of the datasets on ozone absorption cross-sections.

\begin{tabular}{lll}
\hline Dataset & $T, \mathrm{~K}$ & Wavelength, nm \\
\hline Barnes and Mauersberger (1987) & $195,221,237,253,273,297,318$ & 253.65 \\
El Helou et al. (2005) & $144,175,223,225,291,293$ & $543.7,594.3,604.8,612.1,633$ \\
& $150,222,294$ & $748.7,779.4,817.2,853.2$, \\
& $150,222,294$ & $898.3,944.3,991.8,1046.8$ \\
Enami et al. (2004) & $215,245,260,273,298$ & 762.07 \\
& $214,245,273,296$ & 764.47 \\
\hline Bogumil et al. (2003) & $203,223,243,273,293$ & $230-1084$ \\
\hline Burkholder and Talukdar (1994) & $220,240,260,280,295$ & $407-762$ \\
\hline Burrows et al. (1999) & $202,221,241,273,293$ & $231-794$ \\
\hline Paur and Bass (BP) (1984) & $203,223,246,276,280$ & $245-340$ \\
& Polynomial coefficients & $245-343$ \\
\hline BMD, Brion et al. (1993, 1998), & 218 & $195-650$ \\
Malicet et al. (1989, 1995) & $228,243,273$ & $195-520$ \\
& 295 & $195-830$ \\
& Polynomial coefficients & $195-520$ \\
\hline Voigt et al. (2001) & $203,223,243,280,293$ & $230-851$ \\
This work & $193-293$ in 10 K step & $213-1100$ \\
\hline
\end{tabular}

only available for temperatures down to $218 \mathrm{~K}$ and for limited spectral regions only. The measurements by Paur and Bass (1984), often abbreviated as BP, were scaled to Hearn's value at $253.65 \mathrm{~nm}$ at room temperature (Hearn, 1961) and in turn used for scaling the broadband low-resolution data by Bogumil et al. (2003). Low-resolution data by Burrows et al. (1999) were measured absolutely using the titration method at $293 \mathrm{~K}$ and scaled at all temperatures assuming invariant integrated optical densities. The integrated absorption cross-sections of Burrows et al. (1999) were later used for absolute scaling of the broadband high-resolution data by Voigt et al. (2001). Measurements by Burkholder and Talukdar (1994) were normalized using the absolute absorption cross-sections of Anderson and Mauersberger (1992) measured at five specific wavelengths near the peak of the Chappuis band at $298 \mathrm{~K}$.

Broadband datasets are available as original experimental data at selected temperatures and as wavelength-dependent temperature coefficients from a quadratic polynomial fit (details will be given below). Liu et al. (2007) derived polynomial coefficients from the BMD data at four temperatures excluding the $273 \mathrm{~K}$ data. Polynomial coefficients obtained from BP measurements are included in HITRAN 2008, a well-known high-resolution transmission molecular absorption database (Rothman et al., 2009).

Absolute cross-section measurements at single wavelengths by El Helou et al. (2005) and Barnes and Mauersberger (1987) were performed at all temperatures using pure ozone, while data by Enami et al. (2004) were scaled using Hearn's value at $253.65 \mathrm{~nm}$.
There is an obvious lack of consistent and consolidated data on $\mathrm{O}_{3}$ absorption cross-sections. The available literature data do not fulfil the criteria of being available for many temperatures including the lowest atmospheric temperatures (below $200 \mathrm{~K}$ ) and having broad spectral coverage from the UV to NIR and sufficient spectral resolution (better than $0.1 \mathrm{~nm}$ in the UV).

This paper is focused on the results on the temperature dependence of our new ozone cross-section measurements in the broad spectral region from $213 \mathrm{~nm}$ to $1100 \mathrm{~nm}$ obtained for temperatures from $293 \mathrm{~K}$ to $193 \mathrm{~K}$ in steps of $10 \mathrm{~K}$. The experimental setup, associated uncertainties and methods for data analysis are briefly discussed in the Sects. 2 and 3; more details can be found in the companion paper (Gorshelev et al., 2014). In Sect. 3, the new data are analysed and compared with the data listed in Table 1 . The assessment of data quality is performed using several approaches: comparison of the band-integrated cross-sections, polynomial temperature parameterization and calculation of the scaling factors and wavelength shifts between different datasets in the Huggins band.

\section{Experimental setup and uncertainties budget}

Our companion paper provides details on the performance of the experimental setups equipped with an echelle spectrometer and Fourier transform spectrometer (FTS), used for measurements in the UV and UV/vis/NIR spectral ranges respectively. It also contains information on the ozone generators, gas supply, cooling systems, and the liquid nitrogen 
trap for preparation of pure ozone for absolute measurements. Absolute measurements were done in the spectral regions with strong absorption (Huggins and Chappuis bands). All other spectra (obtained from relative measurements in an ozone-oxygen flow) were scaled and concatenated to the absolute spectra separately at each temperature. Relative spectra at $213-310 \mathrm{~nm}$ and $350-450 \mathrm{~nm}$ were recorded using the echelle spectrometer, which is a cross-dispersion instrument with spectral resolution of $0.02 \mathrm{~nm}$ in the $213-450 \mathrm{~nm}$ spectral region. Relative measurements were also performed for $335-350 \mathrm{~nm}$ and $780-1100 \mathrm{~nm}$ using the FTS with a spectral resolution of 1 and $2 \mathrm{~cm}^{-1}$ respectively. Cross-sections were calibrated individually at each temperature, using absolute broadband measurements at $310-335 \mathrm{~nm}$ and $450-780 \mathrm{~nm}$, recorded with the FTS at $1 \mathrm{~cm}^{-1}$ spectral resolution. This way, the new data are independent for every temperature and free from uncertainties of previous studies.

For accurate investigation of the temperature dependence, measurements were performed at eleven temperatures from $293 \mathrm{~K}$ down to $193 \mathrm{~K}$ in $10 \mathrm{~K}$ steps. Gas temperature in the absorption cells was stabilized by continuous flow of ethanol provided by the cryostats (Haake CT $90 \mathrm{~W}$ Phoenix 2). The cells are identical cylindrical double-jacketed quartz vessels having a total length of $140 \mathrm{~cm}$ and an inner diameter of $5 \mathrm{~cm}$. For relative measurements performed at gas flow regimes, a system was equipped with pre-cooling units to assure efficient cooling of the oxygen-ozone mixture, which spent only limited time in the absorption cells. The pre-cooler is a $10 \mathrm{~m}$-long copper pipe fitted into the Haake thermostat vessel. The length of the pipeline exposed to ambient air is approximately $50 \mathrm{~cm}$. Such an arrangement, with proper thermal insulation, reduces ozone-oxygen mixture heating on its way from the cooler to the cell. In order to suppress the copper's catalytic activity to destroy ozone, the inner surface of the pipe was passivated.

The cryostats were capable of limiting the statistical fluctuations to less than $\pm 0.3 \mathrm{~K}$ for all temperatures. The calibration of the internal sensors of the thermostats was verified using several alcohol thermometers. An agreement to within $1 \mathrm{~K}$ or better was found over the full range of operations. Several Pt sensors were used to assess the temperature homogeneity inside the cell and in the cell jacket, which was found to be within $1 \%$. Homogeneity of the cell temperature was within $1 \%$. To exclude possible offsets of the Pt sensors due to contact with the cold surrounding walls, the gas temperature was observed by means of an independent spectroscopic non-intrusive method using the absorption by the oxygen Aband at $760 \mathrm{~nm}$. This method is based on the comparison of measured $\mathrm{O}_{2}$ absorption spectrum with synthetic spectra calculated for different temperatures using line parameters from HITRAN 2008. The algorithm involves an iterative fitting of the rotational structure of the A-band using the dependence of line intensities on rotational temperature. The output is the mean over the cell temperature. The uncertainty of the
HITRAN parameters is about $1 \%$, and the signal-to-noise of the oxygen absorption spectrum was better than $1 \%$.

The Pt sensors, the cryostat internal sensor, and spectroscopic measurements both in the static and flow regime were found to agree within $1-3 \mathrm{~K}$ for almost all temperatures, resulting in an uncertainty of $0.3-1.5 \%$ for the whole range of the temperatures. The rotational temperature is shown in Fig. 1 as a function of the temperature measured by the cryostat sensor. A relative uncertainty of $1.5 \%$ can be taken as the upper limit for the temperature-related uncertainty in the absolute measurements (also shown in Fig. 1 for convenience). For all absolute absorption measurements, ozone concentration was derived using the temperature measured by the cryostat internal sensor.

The uncertainty budget is shown in Table 2. For most of the spectral regions, the largest contribution to the total uncertainty is due to the determination of the absolute ozone concentration. In our quartz cell the ozone concentration was found to fall by $10 \%$ after $10-12 \mathrm{~h}$ at room temperature, and at temperatures below $243 \mathrm{~K}$ the decay rate is lower by at least one order of magnitude. Ozone decay in the cell during gas accumulation before measurements and during spectral recording had to be taken into account only for temperatures above $243 \mathrm{~K}$. The uncertainty of the ozone pressure is about $1 \%$ and better, improving towards lower temperatures.

During the absolute measurements, light source stability was $0.2 \%$ and $2 \%$ for Chappuis and Huggins bands respectively. The uncertainty of the absolute calibration applies as well to the relative spectra that were concatenated. For the majority of the spectral regions, the statistical uncertainty of the absorption measurements is well below $1 \%$; however, in the spectral regions with weak absorption $(350-450 \mathrm{~nm}$ and longer than $900 \mathrm{~nm}$ ) statistical uncertainty can reach up to $30 \%$. More details on the uncertainties, spectral limits of concatenated regions and corresponding measured optical densities can be found in our companion paper (Gorshelev et al., 2014).

\section{Results and discussion}

The absorption cross-sections from this work are presented in Fig. 2. The data cover the spectral region $213-1100 \mathrm{~nm}$ and span over more than seven orders of magnitude. We analysed the absorption cross-sections of $\mathrm{O}_{3}$ at different temperatures by

- determining scaling factors between the new and published band-integrated cross-sections;

- comparing our results and published datasets at wavelengths of the Hg lamp $(254 \mathrm{~nm})$ and those used routinely in ozone remote sensing;

- determining scaling factors and wavelength shifts in the Hartley, Huggins and Chappuis bands with respect 
Table 2. Uncertainty associated with the absorption cross-section obtained from absolute measurements at 50 mbar and 193-293 K and path lengths of 135 and $270 \mathrm{~cm}$ in the Huggins and Chappuis bands.

\begin{tabular}{ll}
\hline \multicolumn{2}{c}{ Systematic uncertainty } \\
\hline Ozone impurity: & \\
$\quad$ oxygen impurity & $0.005 \%$ \\
leaks & $<0.1 \%$ \\
Uncertainty of pressure sensors & $0.5 \%$ \\
Temperature measurements & $<0.3-1.6 \%(1-3 \mathrm{~K})$ \\
Temperature non-uniformity in the system & $<0.3 \%(1 \mathrm{~K})$ \\
Cell length & $<0.04-0.07 \%(<1 \mathrm{~mm})$ \\
\hline Total & $<1.2-2.6 \%$, depending on temperature \\
\hline & Statistical uncertainty \\
\hline Ozone decay & $<1 \%$ \\
Pressure fluctuations & $<0.08 \%(<0.04 \mathrm{mb})$ \\
Temperature fluctuations & $<0.1-0.16 \%(<0.3 \mathrm{~K})$ \\
Light source stability & \\
(relative to optical density OD =1) & $0.2 \%($ vis $) / 2 \%(\mathrm{UV})$ \\
\hline Total & $<1-2.2 \%$, depending on spectral region \\
\hline
\end{tabular}

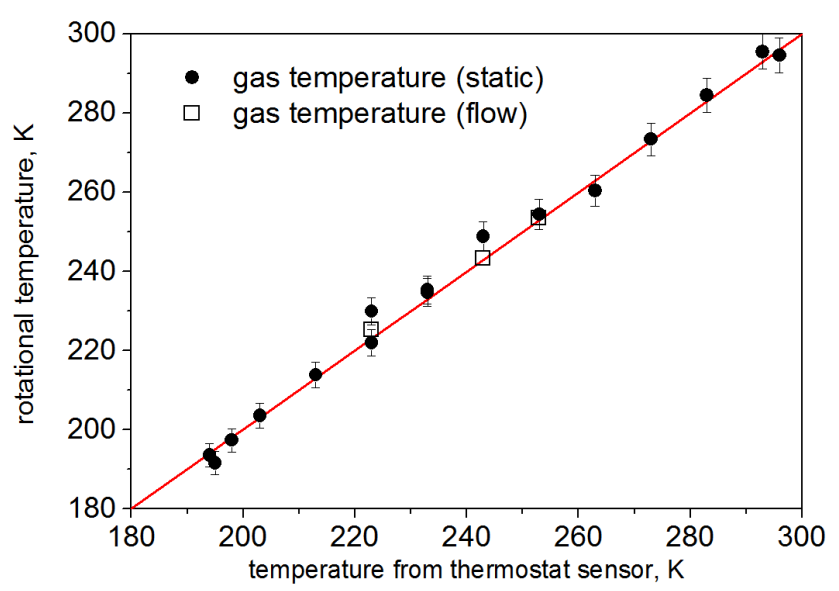

Fig. 1. Correlation between the rotational temperature derived from the oxygen A-band and the temperature obtained using cryostat sensor (symbols).

to the high-resolution BMD and BP datasets and lowresolution data obtained by Bogumil et al. (2003).

\subsection{Band-integrated cross-section comparison}

Band-integrated absorption cross-sections are convenient for comparison of datasets with different spectral resolution. When available, considered cross-sections were integrated using the spectral regions suggested by Orphal (2003): 245$340 \mathrm{~nm}$ in Hartley band, 325-340 nm in Huggins band, and $410-690 \mathrm{~nm}$ in Chappuis band. For the integration in the Wulf band we used the range $663-1000 \mathrm{~nm}$ suggested by Banichevich et al. (1993) from theoretical studies of the

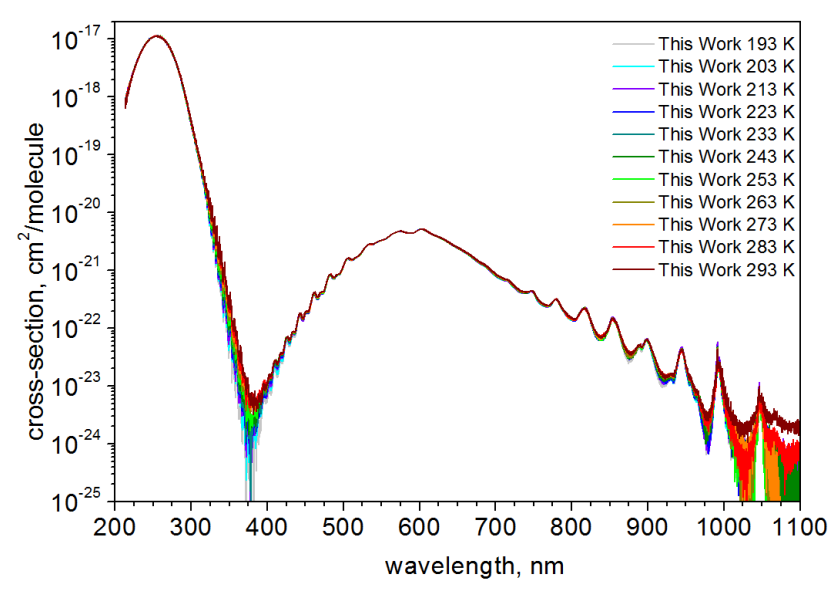

Fig. 2. Overview of our new ozone cross-section data in the $\mathrm{UV} / \mathrm{vis} / \mathrm{NIR}$ measured at 11 temperatures ranging from $193 \mathrm{~K}$ to $293 \mathrm{~K}$.

potential energy surfaces of ozone in its ground and the lowest-lying eight excited states.

Table 3 contains integrated cross-sections calculated using the data obtained in this study, high-resolution BMD and BP datasets, and satellite FM datasets. It also contains the mean values obtained by Orphal (2003) by averaging over several data: BP, BMD, Burrows et al. (1999), Bogumil et al. (2003), Voigt et al. (2001) and Burkholder and Talukdar (1994). Among these datasets only two were measured absolutely: BMD and Burrows et al. (1999). This table also contains ratios of the published datasets over our new dataset.

Integrated cross-sections in the Hartley band agree to within $1 \%$ or better for all considered datasets. In the 
Table 3. Integrated cross-sections, and their ratios (in brackets) to our new dataset.

\begin{tabular}{|c|c|c|c|c|c|}
\hline Dataset/T & $295 \pm 3 \mathrm{~K}$ & $273 \pm 3 \mathrm{~K}$ & $243 \pm 3 \mathrm{~K}$ & $221 \pm 3 \mathrm{~K}$ & $203 \pm 1 \mathrm{~K}$ \\
\hline \multicolumn{6}{|c|}{ Hartley $(245-340 \mathrm{~nm}), \times 10^{-16} \mathrm{~cm}^{2}$ molecule ${ }^{-1} \mathrm{~nm}$} \\
\hline $\begin{array}{l}\text { BMD, Brion et al. }(1993,1998) \text {, } \\
\text { Malicet et al. }(1989,1995)\end{array}$ & $3.52(1)$ & - & $3.50(0.99)$ & $3.49(0.99)$ & - \\
\hline Paur and Bass (1984) & $3.55(1.01)$ & $3.54(1)$ & $3.54(1)$ & $3.53(1)$ & - \\
\hline Guer et al. (2005), Chehade et al. (2013) & $3.56(1.01)$ & 3.56 & 3.56 & 3.56 & 3.56 \\
\hline Bogumil et al. (2003) & $3.56(1.01)$ & $3.55(1.01)$ & $3.54(1)$ & $3.53(1)$ & $3.53(1)$ \\
\hline Burrows et al. (1999) & $3.57(1.01)$ & $3.57(1.01)$ & $3.57(1.01)$ & $3.56(1.01)$ & $3.56(1.01)$ \\
\hline Orphal (2003) & $3.55(1.01)$ & $3.55(1.01)$ & $3.54(1)$ & $3.53(1)$ & $3.53(1)$ \\
\hline This work & 3.53 & 3.53 & 3.53 & 3.54 & 3.52 \\
\hline \multicolumn{6}{|c|}{ Huggins $(325-340 \mathrm{~nm}), \times 10^{-20} \mathrm{~cm}^{2}$ molecule ${ }^{-1} \mathrm{~nm}$} \\
\hline $\begin{array}{l}\text { BMD, Brion et al. }(1993,1998) \text {, } \\
\text { Malicet et al. }(1989,1995)\end{array}$ & $8.30(1)$ & $7.12(0.96)$ & $6.16(0.98)$ & $5.74(0.99)$ & - \\
\hline Paur and Bass (1984) & $8.20(0.99)$ & $7.27(0.98)$ & $6.21(0.99)$ & $5.65(0.98)$ & $5.44(1)$ \\
\hline Guer et al. (2005) & $8.40(1.01)$ & $7.58(1.02)$ & $6.59(1.04)$ & $6.15(1.06)$ & $5.91(1.06)$ \\
\hline Chehade et al. (2013) & $8.20(0.99)$ & $7.38(1)$ & $6.41(1.02)$ & $5.97(1.03)$ & $5.73(1.05)$ \\
\hline Bogumil et al. (2003) & $8.31(1)$ & $7.46(1.01)$ & $6.24(0.99)$ & $5.93(1.03)$ & $5.58(1.02)$ \\
\hline Burrows et al. (1999) & $8.33(1)$ & $7.69(1.04)$ & $6.38(1.02)$ & $5.94(1.03)$ & $5.69(1.04)$ \\
\hline Orphal (2003) & $8.30(1)$ & $7.42(1)$ & $6.30(1)$ & $5.89(1.02)$ & $5.64(1.03)$ \\
\hline This work & 8.29 & 7.41 & 6.28 & 5.77 & 5.46 \\
\hline \multicolumn{6}{|c|}{ Chappuis $(410-690 \mathrm{~nm}), \times 10^{-19} \mathrm{~cm}^{2}$ molecule ${ }^{-1} \mathrm{~nm}$} \\
\hline $\begin{array}{l}\text { BMD, Brion et al. }(1993,1998) \text {, } \\
\text { Malicet et al. }(1989,1995)\end{array}$ & $6.29(1.01)$ & - & - & - & - \\
\hline Guer et al. (2005) & $6.34(1.02)$ & $6.33(1.02)$ & $6.30(1.02)$ & $6.30(1.02)$ & $6.28(1.02)$ \\
\hline Chehade et al. (2013) & $6.34(1.02)$ & $6.33(1.02)$ & $6.29(1.02)$ & $6.23(1.01)$ & $6.28(1.02)$ \\
\hline Bogumil et al. (2003) & $6.40(1.03)$ & $6.42(1.04)$ & $6.35(1.03)$ & $6.32(1.03)$ & $6.41(1.05)$ \\
\hline Burrows et al. (1999) & $6.45(1.04)$ & $6.58(1.06)$ & $6.44(1.05)$ & $6.54(1.06)$ & $6.61(1.08)$ \\
\hline Orphal (2003) & $6.38(1.03)$ & $6.44(1.04)$ & $6.35(1.03)$ & $6.35(1.03)$ & $6.48(1.06)$ \\
\hline This work & 6.22 & 6.2 & 6.16 & 6.15 & 6.13 \\
\hline \multicolumn{6}{|c|}{ Wulf $(663-1000 \mathrm{~nm}), \times 10^{-19} \mathrm{~cm}^{2}$ molecule ${ }^{-1} \mathrm{~nm}$} \\
\hline Bogumil et al. (2003) & $1.099(1.06)$ & $1.08(1.05)$ & $1.06(1.05)$ & $1.044(1.04)$ & $1.06(1.07)$ \\
\hline This work & 1.040 & 1.030 & 1.009 & 1.003 & 0.988 \\
\hline
\end{tabular}

Huggins band, data agree within $0.1-6 \%$, with less agreement towards lower temperatures and at $273 \mathrm{~K}$. In the Chappuis and Wulf bands the new data are lower than the published datasets.

The agreement of our new data with BMD is within $2 \%$ for all values except for the Huggins band at $273 \mathrm{~K}$. The Bogumil et al. (2003) dataset exceeds our new data by about $3-5 \%$ for all temperatures in all bands. In the Hartley and Chappuis bands, our data are only slightly lower (1-2\%) than the broadband dataset obtained by Guer et al. (2005) and recently revised by Chehade et al. (2013). The new experimental data deviate from the mean values obtained by Orphal by about 1-3\% in the Hartley and Huggins bands and up to $6 \%$ in the Chappuis band. The later is partly due to the low absorption in the region near the ozone absorption minimum (near $380 \mathrm{~nm}$ ), which is overestimated by most of the datasets except for BMD and this work. This is particularly evident at lower temperatures.

\subsection{Temperature dependence in the Hartley band}

The temperature dependence in the Hartley band was investigated in many studies, including the works mentioned above. The absorption by the Hartley band system was found to shift towards longer wavelength with increasing temperature due to vibrational excitation, resulting in a redistribution of the vibrational state populations in the ground state (Baiamonte et al., 1966). We fitted a Gaussian profile to the Hartley band between the shortest wavelength available and $300 \mathrm{~nm}$ and analysed the centre position of the Gaussian profile as a function of temperature for several datasets. We found that the Gaussian profile undergoes a shift of $0.14 \mathrm{~nm}$ from $203 \mathrm{~K}$ to $293 \mathrm{~K}$, while BP and Bogumil et al. (2003) data experience a shift of $0.18 \mathrm{~nm}$ and $0.05 \mathrm{~nm}$ respectively for the same temperature change. The BMD data reveal a more pronounced shift of $0.36 \mathrm{~nm}$ from $218 \mathrm{~K}$ to $295 \mathrm{~K}$.

Different groups reported a weak increase of crosssections of about $1 \%$ for temperature change from 295 to 
$229 \mathrm{~K}$ in the Hartley band (Barnes and Mauersberger, 1987; Malicet et al., 1989; Paur and Bass, 1984). Our new data also show a weak temperature dependence (about $2 \%$ in the range 193-293 K). Figure 3 shows our cross-section spectra at $193 \mathrm{~K}$ and $293 \mathrm{~K}$ near $250 \mathrm{~nm}$ and the temperature dependence of various datasets, including ours at $253.65 \mathrm{~nm}$, corresponding to the Hg lamp line. There is a very good agreement between new and BMD data, while the Bogumil et al. (2003) dataset is higher than the new dataset by about $2 \%$. The Bogumil et al. (2003) dataset does not show any clear temperature dependence, while the absorption cross-sections reported in this study slightly increase with decreasing temperature.

\subsection{Temperature dependence in the Huggins band}

The new cross-section data at eleven temperatures in the Huggins band are shown in Fig. 4a. The band contains the spectral window (325-335 nm) widely used for the ozone total column retrievals with the DOAS technique (ColdeweyEgbers et al., 2005) and spectral channels of the Dobson and Brewer spectrophotometers (Fig. 4).

\subsubsection{Polynomial parameterization}

The Bass-Paur parameterization empirically describes the temperature dependence of the cross-sections in the DOAS window as a second-order polynomial, making it possible to interpolate between temperatures (Paur and Bass, 1984). A commonly used equation for the temperature dependence of the cross-section is

$\sigma(\lambda)=10^{-20} \cdot\left[C_{0}(\lambda)+C_{1}(\lambda) \cdot T+C_{2}(\lambda) \cdot T^{2}\right]$,

where $T$ is the temperature in degrees Celsius; $C_{0}, C_{1}$ and $C_{2}$ are wavelength-dependent fitting coefficients; and the crosssection unit is $\mathrm{cm}^{2}$ molecule ${ }^{-1}$.

We analysed the temperature dependence of the new ozone absorption cross-section in the spectral channels of two ground-based instruments: Dobson and Brewer spectrophotometers (Scarnato et al., 2009). The channels fall on maxima and minima of the spectral features in the region 305$340 \mathrm{~nm}$ (wavelengths are indicated in Fig. 4a). For a rough resolution matching with the instrumental slit functions, we convolved our new dataset to $1 \mathrm{~nm}$ and $0.4 \mathrm{~nm}$ using a rectangular slit function for analysis at Dobson and Brewer wavelengths respectively. In addition, we investigated the temperature behaviour at initial spectral resolution of about $0.02 \mathrm{~nm}$ at $328 \mathrm{~nm}$ and $330 \mathrm{~nm}$, which are local absorption minimum and maximum.

The deviations of the new cross-sections from the polynomial fits at wavelengths described above are shown in Fig. 4b (maximal deviations are given in brackets). The quadratic polynomial (Eq. 1) describes the temperature dependence for most wavelengths to within $1.5 \%$, which is within the experimental uncertainty. Generally, wavelengths near ozone
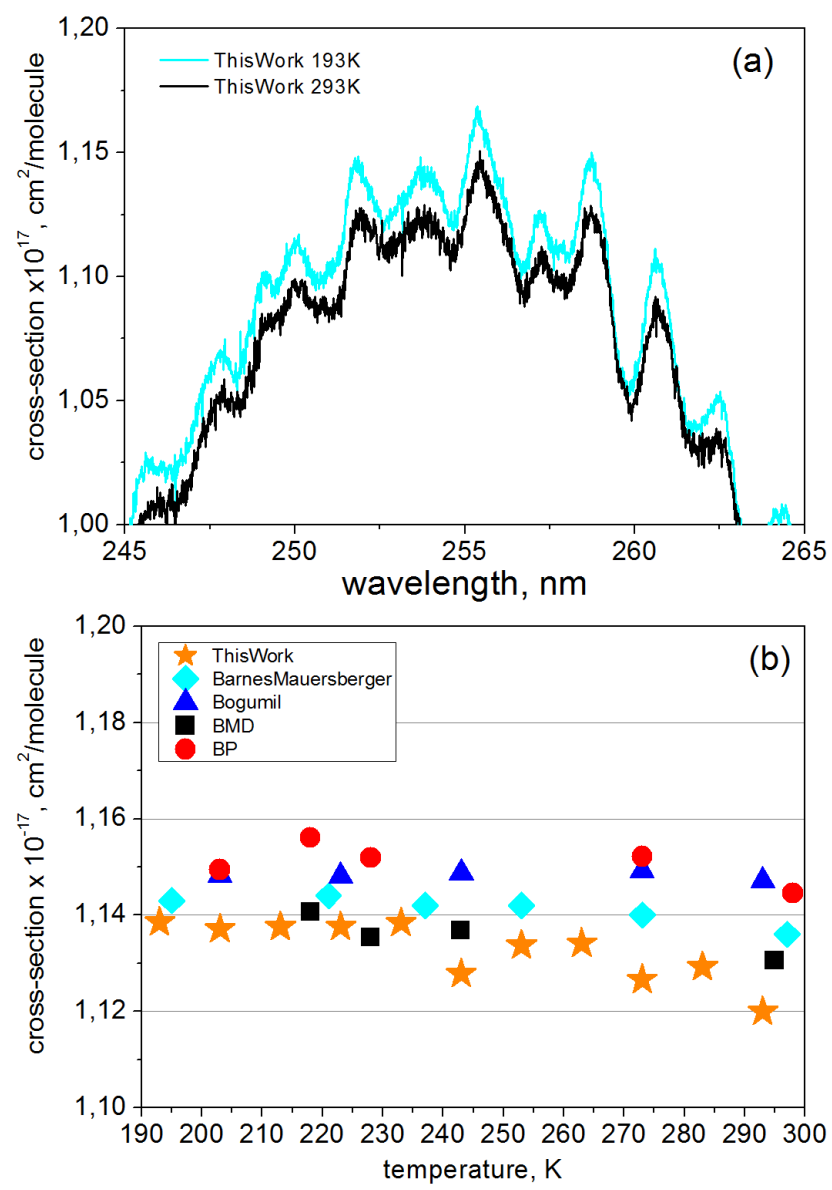

Fig. 3. Temperature dependence of the new experimental crosssections in the UV region: (a) cross-sections for $293 \mathrm{~K}$ (dark line) and $193 \mathrm{~K}$ (light line); (b) temperature dependence at $253.65 \mathrm{~nm}$. Stars - new experimental data; diamonds - Barnes and Mauersberger (1987); triangles - Bogumil et al. (2003); squares - BMD; circles $-\mathrm{BP}$.

absorption maxima exhibit smaller deviations. Figure 5 (upper panel) shows the difference between the experimental data and calculated data for two temperatures: $193 \mathrm{~K}$ and 293 K. Deviations are generally less than $2 \%$. In principle, calculated data have better signal-to-noise level compared to the experimental ones, as artefacts from measurements at a single temperature are smoothed out.

Differences between coefficients $C_{0}(\lambda)$ obtained in several works are shown in the lower panel in Fig. 5. We analysed data from this work and those obtained by Bass and Paur from their measurements in the range $203-298 \mathrm{~K}$ and by Liu et al. (2007) using BMD measurements at 218, 228, 243 and 295 K. Deviations are within $2 \%$ in the Huggins band but increase at longer wavelengths. BP data become noisier above $335 \mathrm{~nm}$. Some of the differences between the coefficients can be explained by slight resolution mismatch and uncertainty in the wavelength calibration in any of the considered datasets. 

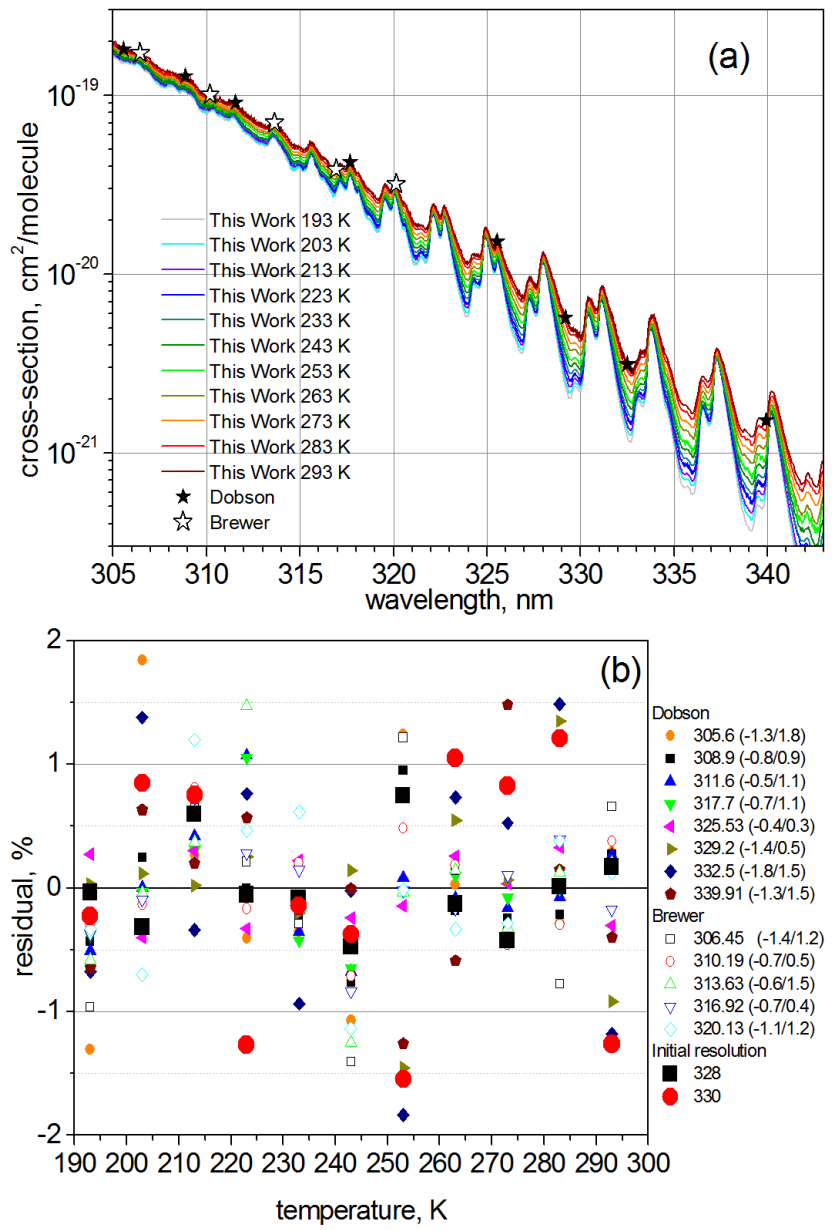

Fig. 4. Temperature dependence of the ozone absorption crosssections: (a) ozone cross-section data in the Huggins band as measured in this study; (b) residuals from the polynomial fit for the temperature dependence in the Huggins band. Small closed and open symbols - Dobson and Brewer spectral channels respectively; big closed squares and circles - data at $328 \mathrm{~nm}$ and $330 \mathrm{~nm}$. Numbers in brackets represent the maximal deviations.

\subsubsection{Comparison with high and low spectral resolution datasets}

We performed detailed comparisons of the new crosssections in the spectral region $325-340 \mathrm{~nm}$ with the highresolution datasets (BMD, BP and Voigt et al., 2001) and the low-resolution Bogumil et al. (2003) dataset. Data were analysed by determining scaling factors and wavelength shifts between the considered datasets and mean differences. Data were interpolated on a common wavelength grid, converted to vacuum wavelength and then shifted and scaled until the smallest mean difference over the spectral region was reached. For comparison with the low-resolution dataset by Bogumil et al. (2003) our new data were convolved with the Gaussian profile with full width at half maximum (FWHM) of $0.2 \mathrm{~nm}$. To check the linearity of the shifts and scaling

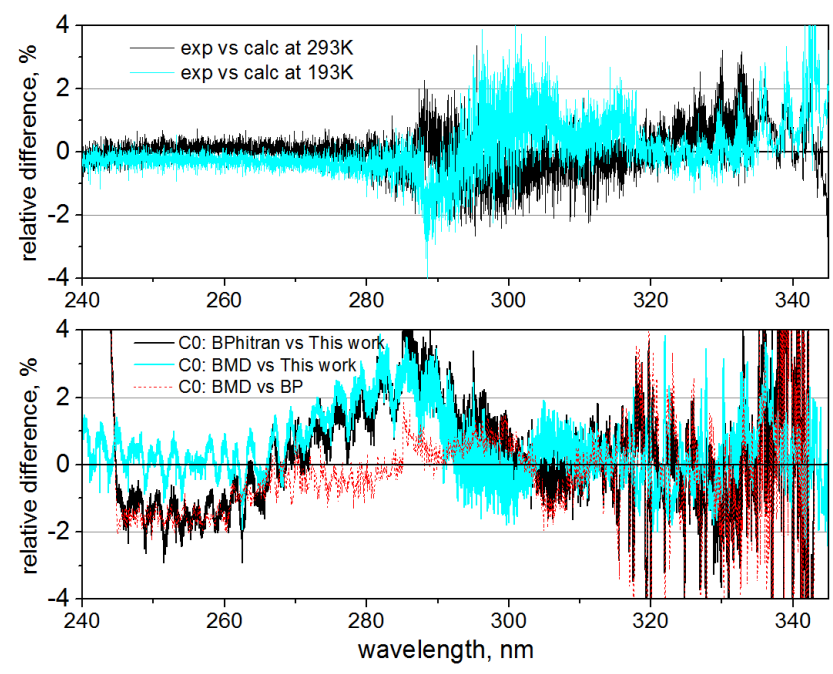

Fig. 5. Temperature dependence of the ozone absorption crosssections. (upper panel) Difference between the experimental and Eq. (1) for 193 and $293 \mathrm{~K}$ (light and dark lines). (lower panel) Difference between fitting coefficients $C_{0}$ in Eq. (1) from this work and BP (dark line) and BMD (light line), and between BP and BMD (dashed line).

factors, we performed the analysis for three different spectral regions (323-330 nm, 332-340 $\mathrm{nm}$ and $323-340 \mathrm{~nm}$ ) and averaged values to obtain uncertainties. More details on the comparison routine are given in our companion paper (Gorshelev et al., 2014).

We compared the experimental BMD data to the temperature parameterized BMD data obtained by Liu et al. (2007) in order to provide an additional independent check for the uncertainty due to the assumption of the quadratic temperature dependence. The parameterized BMD spectrum deviates from the experimental BMD within the experimental accuracy limits both in absolute values and shifts. Mean difference in the spectral region $325-340 \mathrm{~nm}$ is about $1 \%$. Therefore, in the following comparisons we used both the original and parameterized cross-sections, which are practical in case of missing data at some temperatures. However, since parameterization itself is a manipulation of the data, we tried to use original BMP and BP datasets as extensively as possible.

We found differences between the parameterized BMD and the parameterized BP datasets that are outside of the accuracy limits reported for both datasets. Wavelength shifts of 0.015-0.02 nm were found. Without shift corrections and scaling, differences are up to $20 \%$ and more due to the sharp features in this spectral region; the mean over the region difference is $2-5 \%$. After correcting for wavelength shifts the mean difference is about $1-3 \%$ with larger deviations at low temperatures.

The comparison of our experimental data with published datasets is shown in Fig. 6. Here wavelength shifts, scaling factors and mean differences in the spectral region 325$340 \mathrm{~nm}$ are depicted at different temperatures. 
Scaling factors and wavelength shifts were applied to the published datasets to match the new dataset. The remaining deviations after scaling and shifting are then due to non-linear spectral/wavelength calibration differences, differences in the instrumental slit functions and resolution mismatch between the datasets. In addition, slight mismatch of temperatures has to be taken into account when comparing original experimental data in this region because of the high sensitivity of the Huggins band spectral features to the temperature. All mean differences are positive in Fig. 6, because absolute values of differences at every point were taken and averaged. This corresponds to the "worst-case scenario" for mean differences, when summands are not compensating each other due to alternating sign (this happens when, for example, within the considered range two cross-section curves are intersecting).

We compared our new data at $213 \mathrm{~K}$ with the experimental BP data obtained at $218 \mathrm{~K}$ and our data at $223 \mathrm{~K}$ with BP data at 218 and $228 \mathrm{~K}$ and found scaling factors of up to $-6 \%$ needed to minimize the differences which are also due to the temperature mismatch of $5 \mathrm{~K}$. We observed wavelength shifts of $0.015-0.02 \mathrm{~nm}$ between the BP and our new data (similar to what have been seen when comparing BP to BMD) and the mean difference of about 2-3\%, increasing to 4-5\% at low temperatures. HITRAN 2008 version accommodates polynomial coefficients obtained for the BP data after wavelength correction performed on the basis of Voigt et al. (2001) measurements. Comparison with the data calculated using these coefficients revealed scaling factors of $1 \%$ for most temperatures and a relatively small wavelength shift $(0.005-0.01 \mathrm{~nm})$.

Duplicate points on Fig. 6 for two temperatures (218 K and $228 \mathrm{~K})$ designate differences between experimental BMD data at those temperatures and new experimental data at adjacent temperatures of $213 \mathrm{~K}, 223 \mathrm{~K}$, and $233 \mathrm{~K}$. We compared our data at 213 and $223 \mathrm{~K}$ to the experimental BMD data at $218 \mathrm{~K}$ and our data at 223 and $233 \mathrm{~K}$ to the BMD data at $228 \mathrm{~K}$ and found scaling factors of $\pm 2 \%$ needed to minimize the differences. Small mean shifts of $0.005 \mathrm{~nm}$ and less are observed for the window 323-340 nm between the new data and experimental BMD. However, real shifts are non-linear because we found different wavelength shifts for smaller regions $323-330 \mathrm{~nm}$ and $332-340 \mathrm{~nm}$. The same was reported by Orphal (2003). The mean relative difference is about $1-2 \%$ at $325-340 \mathrm{~nm}$, which is within the experimental accuracy limits. Calculated BMD data agree with our new experimental data within accuracy limits both with respect to absolute values and spectral shifts. Small spectral shifts were found between our new data and the Voigt et al. (2001) and Bogumil et al. (2003) data. Bogumil et al. (2003) and Voigt et al. (2001) data exceed the new data by $1-4 \%$.

At wavelengths shorter than $325 \mathrm{~nm}$, wavelength shift seems to be less important; however, it still influences the agreement between the BP and other data. BP data at low temperature deviate both from BMD and the new data. In
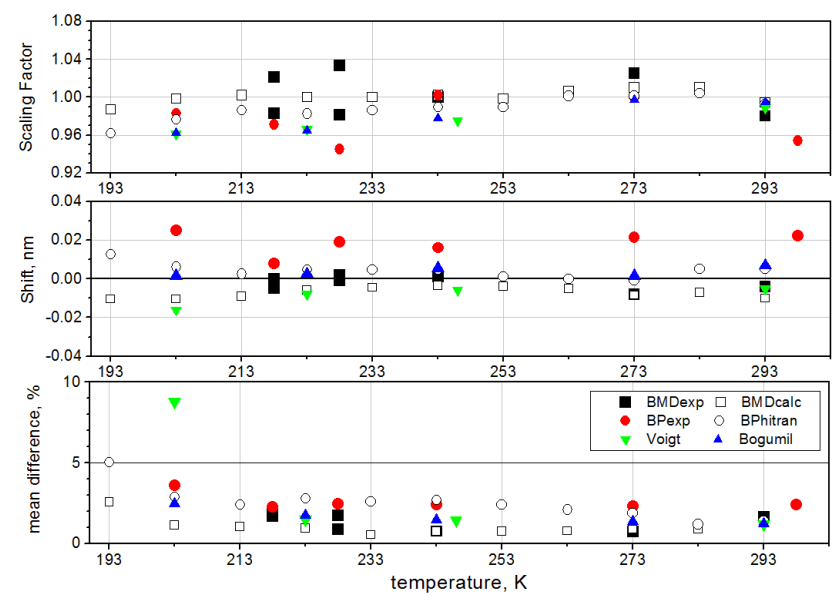

Fig. 6. Comparison of the results from this study with BP, BMD, Voigt et al. (2001) and Bogumil et al. (2003) in the window 325$340 \mathrm{~nm}$ (for more details, please read the text): (upper panel) scaling factors (to match other data to new data); (middle panel) wavelength shift (to match other data to new data); (lower panel) relative mean difference (other data after applying corrections for shifts and scaling factors minus new data). Filled symbols - experimental data; open symbols - data obtained from the polynomial parameterization (Eq. 1). Squares - BMD, circles - BP, triangles - Voigt et al. (2001) and Bogumil et al. (2003) datasets.

the 315-325 nm region, the mean difference between the parameterized $\mathrm{BMD}$ and $\mathrm{BP}$ datasets is 1.4 to $5.7 \%$ depending on the temperature. Agreement can be slightly improved (mostly for room temperature) to $0.6-5.4 \%$ using wavelength shifts. New data differ from BMD and BP data by $0.9-$ $1.9 \%$ and $1.5-5.4 \%$ respectively, with stronger deviations corresponding to the data at low temperature. When using wavelength correction, only insignificant improvement can be achieved: down to $0.7-1.7 \%$ and $0.7-5 \%$ for BMD and BP respectively. During this correction, wavelength shifts of $0.015-0.03 \mathrm{~nm}$ (depending on temperature) were applied to BP to match the new data, and a shift of about $0.008 \mathrm{~nm}$ (almost independent of the temperature) applied to BMD to match the new data. A small scaling correction of about $1.5 \%$ further decreases mean difference between the BMD and the new data down to $0.3-1.4 \%$.

\subsection{Temperature dependence in Chappuis and Wulf bands}

\subsubsection{Absorption region $350-450 \mathrm{~nm}$}

Few experimental data are available in the weak absorption region between the Huggins and Chappuis absorption bands $(\sim 375 \mathrm{~nm})$. The BP dataset does not cover this region. Figure 7 shows our results as well as BMD and Bogumil et al. (2003) data in this region. The BMD experimental data are only available down to $218 \mathrm{~K}$; for all temperatures below that, Bass-Paur temperature parameterization must be used. 

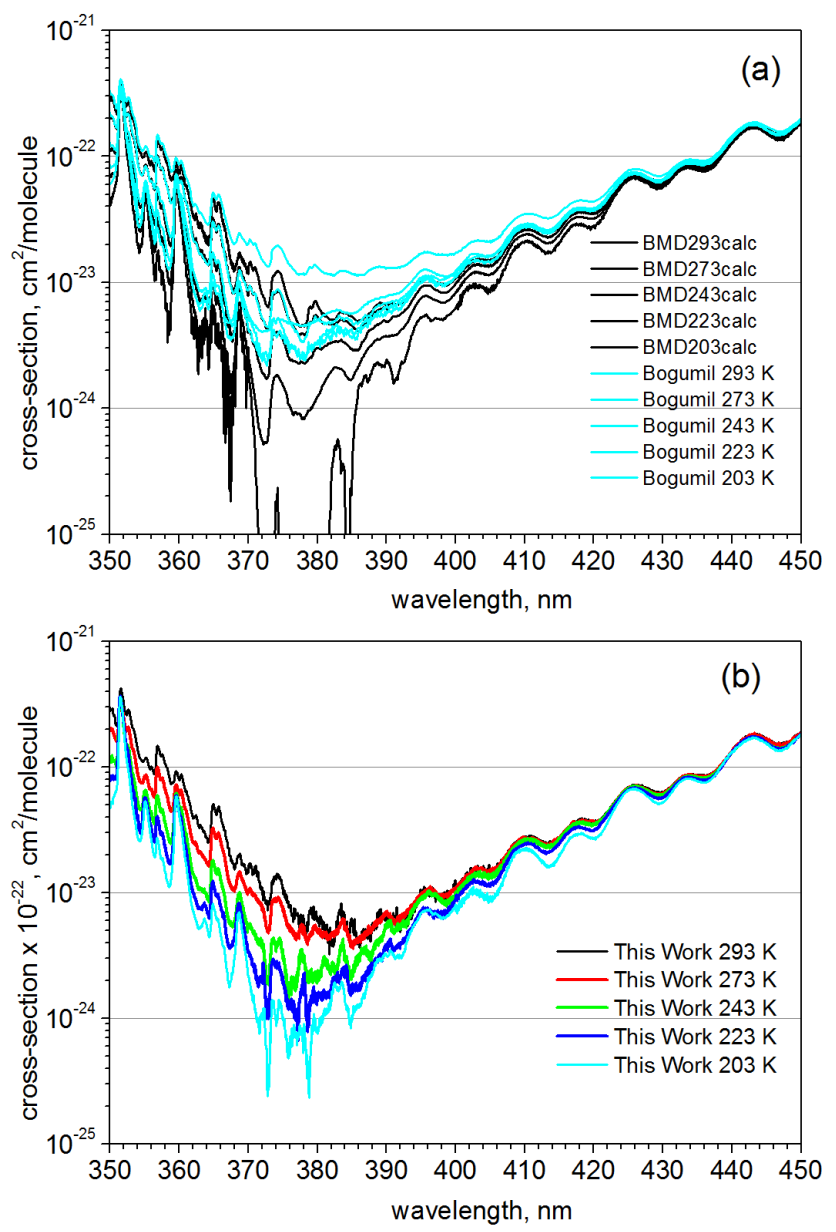

Fig. 7. Ozone cross-sections at $350-450 \mathrm{~nm}$ at different temperatures: (a) Bogumil et al. (2003) and BMD; (b) this work.

Cross-sections calculated using temperature coefficients obtained by Liu et al. (2007) from the BMD experimental data drastically fall at $203 \mathrm{~K}$. The low-resolution dataset by Bogumil et al. (2003) overestimates the minimum absorption. Near the bottom of the weak absorption region (370$390 \mathrm{~nm}$ ), our new data appear to be noisier than the BMD dataset. We expect improvement of this region from our future measurements.

\subsubsection{Visible region $450-700 \mathrm{~nm}$}

Very weak temperature dependence was observed in our new measurements around $600 \mathrm{~nm}$ (about $1 \%$ between $193 \mathrm{~K}$ and $293 \mathrm{~K}$ ), with a small increase of cross-sections with decreasing temperatures.

Only a few studies have been performed below $293 \mathrm{~K}$ in the Chappuis (Fig. 8) and Wulf (Fig. 9) bands, especially in the NIR region. The most extensive dataset obtained so far was reported by Bogumil et al. (2003). In the Chappuis band and NIR, our new data are lower than the Bogumil et al. (2003) dataset (2-4.5\% in the region $540-850 \mathrm{~nm})$ and agree very well in the region $540-630 \mathrm{~nm}$ with the data of
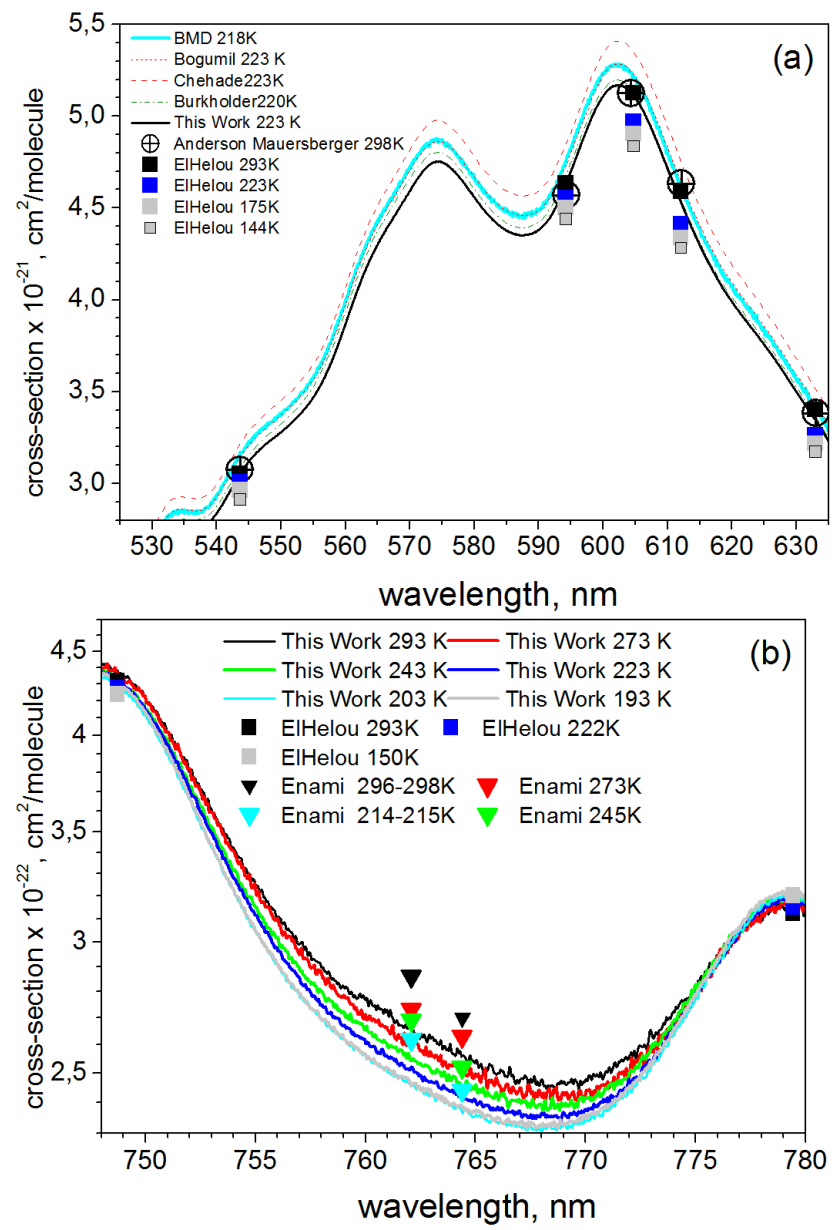

Fig. 8. Ozone cross-sections in the Chappuis and Wulf bands at different temperatures: (a) near maximum of the Chappuis band (squares - El Helou et al. (2005); circles - Anderson and Mauersberger (1992); red dashed line - Bogumil et al. (2003) and Chehade et al. (2013); light-blue solid lines - BMD; dash-dot line - Burkholder and Talukdar (1994); black solid lines - this work); (b) near $760 \mathrm{~nm}$ (squares - El Helou et al. (2005); triangles - Enami et al. (2004); lines - this work).

Burkholder and Talukdar (1994) (0.4-1\%), which have reported uncertainty of $1 \%$ and better for wavelengths longer than $450 \mathrm{~nm}$. There is a good agreement between our data and the data of El Helou et al. (2005); however, the latter show a reversed temperature dependence around $600 \mathrm{~nm}$. In Table 4, several datasets are compared at discrete wavelengths taken from El Helou et al. (2005).

High spectral resolution BMD data in the Chappuis band are only available for $218 \mathrm{~K}$. Our data are lower by $2-3 \%$. BMD report a systematic uncertainty of $1.5 \%$ and random uncertainty of $0.9-2 \%$ in the visible/IR region. Therefore, there is a good agreement between BMD and our data.

In the 540-630 nm region our data are slightly lower (1$2.5 \%$ ) than the GOME-2 FM3 data obtained by Guer et al. (2005) and the recently revised GOME-2 FM3 data by Chehade et al. (2013). 
Table 4. Comparison of some ozone cross-section datasets at low temperatures in the Chappuis and Wulf bands, $\times 10^{-22} \mathrm{~cm}^{2} \mathrm{molecule}-1$.

\begin{tabular}{lrrrrrr}
\hline $\begin{array}{l}\text { Wavelength, } \\
\mathrm{nm}\end{array}$ & $\begin{array}{r}\text { El Helou et al. (2005) } \\
\text { 222-223 K }\end{array}$ & $\begin{array}{r}\text { Burkholder and Talukdar } \\
(1994), 220 \mathrm{~K}\end{array}$ & $\begin{array}{r}\text { Bogumil et al. } \\
(2003), 223 \mathrm{~K}\end{array}$ & $\begin{array}{r}\text { BMD, Brion et al. (1993, 1998), } \\
\text { Malicet et al. (1989, 1995), 218 K }\end{array}$ & $\begin{array}{r}\text { This work, } \\
223 \mathrm{~K}\end{array}$ \\
\hline 543.667 & 30.05 & - & 30.850 & 31.418 & 31.573 & 30.530 \\
594.261 & 45.53 & - & 46.780 & 47.376 & 47.633 & 46.335 \\
604.78 & 49.7 & - & 51.301 & 52.247 & 52.067 & 51.101 \\
612.14 & 44.12 & - & 45.412 & 46.370 & 46.126 & 45.259 \\
632.991 & 32.63 & - & 33.645 & 34.314 & 34.154 & 33.462 \\
748.721 & 4.271 & 4.235 & 4.037 & 4.464 & - & 4.328 \\
779.416 & 3.149 & 3.199 & 1.541 & 3.328 & - & 3.181 \\
817.224 & 2.211 & 2.314 & - & 2.335 & - & 2.230 \\
853.234 & 1.495 & 1.577 & - & 1.573 & - & 0.617 \\
898.247 & 0.654 & 0.667 & - & 0.686 & - & 0.420 \\
944.259 & - & 0.466 & - & 0.471 & - & 0.469 \\
991.841 & - & 0.609 & - & 0.450 & - & 0.076 \\
1046.766 & - & 0.128 & - & 0.0693 & & -10 \\
\hline
\end{tabular}

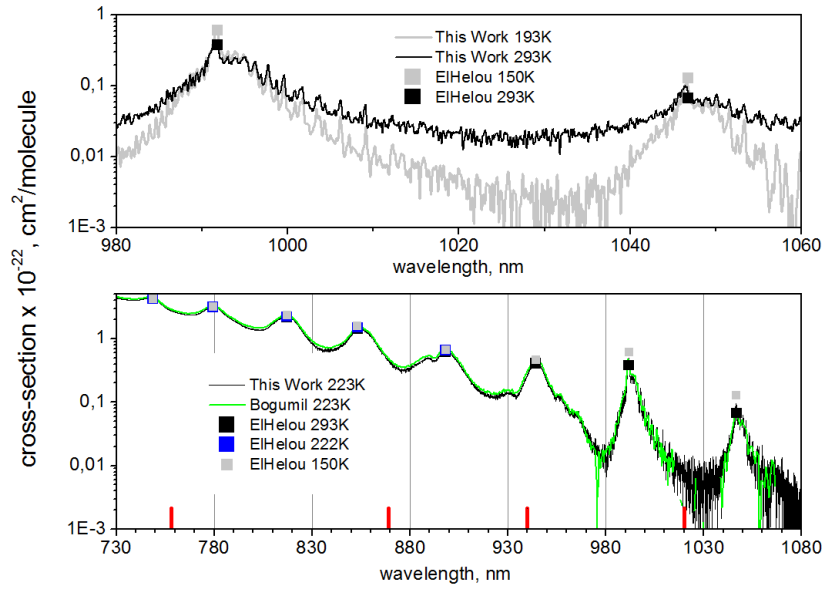

Fig. 9. Ozone cross-sections in the Wulf band at different temperatures. Lines - new data and Bogumil et al. (2003); squares - El Helou et al. (2005). The spectral channels from SAGE II are indicated by short vertical lines.

\subsubsection{NIR region $700-1100 \mathrm{~nm}$}

The region around $760 \mathrm{~nm}$ contains an absorption band of oxygen, which is visible in the Bogumil et al. (2003) dataset due to the oxygen-ozone mixture used during the measurements. The Bogumil et al. (2003) data do not show any clear temperature dependence. The new dataset measured using pure ozone is free of oxygen absorption and shows a clear temperature dependence (Fig. 8b). Absolute values are lower by a few percent (3-4\%) compared to Bogumil et al. (2003). The data of Enami et al. (2004) show similar temperature dependence, but cross-sections are higher than the new data (about 2-3\%).

There is good agreement in the NIR spectral region between this study and the data of Bogumil et al. (2003) and El Helou et al. (2005), both in absolute values and in the temperature dependence. Measurements of El Helou et al. (2005) performed at $150 \mathrm{~K}$ are only slightly different from those at $223 \mathrm{~K}$.

Temperature dependence in the NIR region around $1000 \mathrm{~nm}$ is somewhat masked by the low signal-to-noise ratio in the Bogumil et al. (2003) dataset. The new dataset reveals a clear temperature dependence, and it resolves the rotational structure (Fig. 9). At wavelengths longer than $1050 \mathrm{~nm}$ our cross-sections drastically drop and have low signal-to-noise ratio. Our FTS setup was not optimized for this spectral region.

\section{Conclusions}

Most of the broadband datasets measured so far for different temperatures were either limited in spectral region or resolution, or were scaled to literature data, thus inheriting uncertainties from previous studies. Our experimental setup made it possible to obtain absolute high-resolution broadband data at various temperatures down to a record low $193 \mathrm{~K}$. The uncertainty of the absolute measurements related to the ozone decay practically disappears at temperatures below $243 \mathrm{~K}$ due to the very slow decay time compared to the typical measurements duration. A spectroscopic method using measurements of the oxygen A-band was used to obtain an independent estimate of the mean gas temperature across the absorption cell in addition to the commonly used contact temperature sensors.

The new data agree within experimental uncertainty with the BMD dataset at all temperatures, excluding the region near $380 \mathrm{~nm}$ at low temperatures (below $1 \%$ in the Hartley band, $1-2 \%$ in the Huggins band and about $2-3 \%$ in the Chappuis band).

The new broadband dataset offers additional information on the temperature dependence of the ozone absorption 
cross-sections, both inside and outside of the previously available temperatures range. Our study confirms that a second-order polynomial reproduces the temperature dependence of the experimental data in the Huggins band within the experimental uncertainty limits. The new dataset obtained at 11 temperatures provides more accurate temperature parameterization compared to that typically derived from 4 to 5 temperature points in the literature data that are currently used in the ozone retrievals.

We found a weak temperature dependence on the top of the Hartley and Chappuis bands, which is however within experimental uncertainties. In the Wulf band we obtain a clear temperature dependence in agreement with low-resolution measurements reported by Bogumil et al. (2003). We plan to investigate temperature dependence in the NIR region in more details using an improved dual-channel FTS setup with higher signal-to-noise ratio. Improved measurements in the thermal IR can also be of interest for comparisons between UV, visible, NIR and thermal IR ozone retrievals in the current and future remote sensing missions. The NIR measurements as well as new measurements near $380 \mathrm{~nm}$ (absorption minimum between Higgins and Chappuis bands) are ongoing.

The new ozone cross-sections are available on the homepage of the Molecular Spectroscopy Laboratory of the Institute of the Environmental Physics, University of Bremen (http://www.iup.uni-bremen.de/gruppen/ molspec/databases/index.html). Cross-section updates from future measurements are expected and will be made available on our homepage.

Acknowledgements. This study was produced within the framework of a project devoted to the harmonization of the satellite cross-section datasets, initiated at the University of Bremen and supported in part by the European Space Agency (within the ESA HARMONICS project), by the State of Bremen, University of Bremen, and DLR.

Edited by: J. Staehelin

\section{References}

Anderson, S. M. and Mauersberger, K.: Laser measurements of ozone absorption cross sections in the Chappuis band, Geophys. Res. Lett., 19, 933-936, 1992.

Baiamonte, V. D., Snelling, D. R., and Bair, E. J.: Vibrational energy of ozone during photolytic explosion, J. Chem. Phys., 44, 673682,1966

Banichevich, A., Peyerimhoff, S. D., and Grein, F.: Potential energy surfaces of ozone in its ground state and in the lowest-lying eight excited states, Chem. Phys., 178, 155-188, 1993.

Barnes, J. and Mauersberger, K.: Temperature dependence of the ozone absorption cross section at the $253.7 \mathrm{~nm}$ Mercury line, J. Geophys. Res., 92, 14861-14864, 1987.
Brion, J., Chakir, A., Daumont, D., Malicet, J., and Parisse, C.: High-resolution laboratory absorption cross section of $\mathrm{O}_{3}$. Temperature effect, Chem. Phys. Lett., 213, 610-612, 1993.

Brion, J., Chakir, A., Charbonnier, J., Daumont, D., Parisse, C., and Malicet, J.: Absorption spectra measurements for the ozone molecule in the $350-830 \mathrm{~nm}$ region, J. Atmos. Chem., 30, $291-$ 299, 1998.

Bogumil, K., Orphal, J., Homann, T., Voigt, S., Spietz, P., Fleischmann, O. C., Vogel, A., Hartmann, M., Bovensmann, H., Frerick, J., and Burrows, J. P.: Measurements of molecular absorption spectra with the SCIAMACHY pre-flight model: instrument characterization and reference data for atmospheric remotesensing in the $230-2380 \mathrm{~nm}$ region, J. Photoch. Photobio. A., 157, 157-167, 2003.

Burkholder, J. B. and Talukdar, R. K.: Temperature Dependence of the Ozone, Geophys. Res. Lett., 21, 581-584, 1994.

Burrows, J. P., Richter, A., Dehn, A., Deters, B., Himmelmann, S., Voigt, S., and Orphal, J.: Atmospheric Remote-Sensing Reference Data from GOME. 2. Temperature-Dependent Absorption Cross Sections of $\mathrm{O}_{3}$ in the 231-794 nm range, J. Quant. Spectrosc. Ra., 61, 509-517, 1999.

Chehade, W., Gür, B., Spietz, P., Gorshelev, V., Serdyuchenko, A., Burrows, J. P., and Weber, M.: Temperature dependent ozone absorption cross section spectra measured with the GOME-2 FM3 spectrometer and first application in satellite retrievals, Atmos. Meas. Tech., 6, 1623-1632, doi:10.5194/amt-6-1623-2013, 2013.

Coldewey-Egbers, M., Weber, M., Lamsal, L. N., de Beek, R., Buchwitz, M., and Burrows, J. P.: Total ozone retrieval from GOME UV spectral data using the weighting function DOAS approach, Atmos. Chem. Phys., 5, 1015-1025, doi:10.5194/acp5-1015-2005, 2005

El Helou, Z., Churassy, S., Wannous, G., Bacis, R., and Boursey, E. Absolute cross sections of ozone at atmospheric temperatures for the Wulf and the Chappuis bands, J. Chem. Phys., 122, 244311, doi:10.1063/1.1937369, 2005.

Enami, S., Ueda, J., Nakano, Y., Hashimoto, S., and Kawasaki, M.: Temperature-dependent absorption cross sections of ozone in the Wulf-Chappuis band at 759-768 nm, J. Geophys. Res., 109, D05309, doi:10.1029/2003JD004097, 2004.

Gorshelev, V., Serdyuchenko, A., Weber, M., Chehade, W., and Burrows, J. P.: High spectral resolution ozone absorption crosssections - Part 1: Measurements, data analysis and comparison with previous measurements around $293 \mathrm{~K}$, Atmos. Meas. Tech., 7, 609-624, doi:10.5194/amt-7-609-2014, 2014.

Guer, B., Spietz, P., Orphal, J., and Burrows, J. P.: Absorption Spectra Measurements with the GOME-2 FMs using the IUP/IFEUB's Calibration Apparatus for Trace Gas Absorption Spectroscopy CATGAS, Final Report, Contract No. 16007/02/NL/SF, ESA/EUMETSAT, Bremen, 2005.

Hearn, A. G.: The absorption of Ozone in the Ultra-violet and Visible Regions of the Spectrum, P. Phys. Soc., 78, 932-940, 1961.

Liu, X., Chance, K., Sioris, C. E., and Kurosu, T. P.: Impact of using different ozone cross sections on ozone profile retrievals from Global Ozone Monitoring Experiment (GOME) ultraviolet measurements, Atmos. Chem. Phys., 7, 3571-3578, doi:10.5194/acp7-3571-2007, 2007 
Malicet, J., Brion, J., and Daumont, D.: Temperature dependence of the absorption cross-section of ozone at $254 \mathrm{~nm}$, Chem. Phys. Lett., 158, 293-296, 1989.

Malicet, J., Daumont, D., Charbonnier, J., Chakir, C., Parisse, A., and Brion J.: Ozone UV Spectroscopy. II: Absorption cross sections and temperature dependence, J. Atmos. Chem., 21, 263273, 1995.

Orphal, J.: A critical review of the absorption cross-sections of $\mathrm{O}_{3}$ and $\mathrm{NO}_{2}$ in the ultraviolet and visible, J. Photoch. Photobio. A., 157, 185-209, 2003.

Paur, R. J. and Bass, A. M.: The Ultraviolet Cross-Sections of Ozone: II. Results and temperature dependence, in: Atmospheric ozone; Proc. Quadrennial Ozone Symposium, edited by: Zeferos, C. S. and Ghazi, A., Halkidiki Greece, 1984, Dordrecht: Reidel, D., 611-615, 1984.

Rothman, L. S., Gordon, I. E., Barbe, A., Benner, C. D., Bernath, P. F., Birk, M., Boudon, V., Brown, L. R., Campargue, A., Champion, J.-P., Chance, K., Coudert, L. H., Dana, V., Devi, V. M., Fally, S., Flaud, J.-M., Gamache, R. R., Goldman, A., Jacquemart, D., Kleiner, I., Lacome, N., Lafferty, W. J., Mandin, J.Y., Massie, S. T., Mikhailenko, S. N., Miller, C. E., MoazzenAhmadi, N., Naumenko, O. V., Nikitin, A. V., Orphal, J., Perevalov, V. I., Perrin, A., Predoi-Crosss, A., Rinsland, C. P., Rotger, M., Šimečkova, M., Smith, M. A. H., Sung, K., Tashkun, S. A., Tennyson, J., Toth, R. A., Vandaele, A. C., and Auwera Vander, J.: The HITRAN 2008 molecular spectroscopic database, J. Quant. Spectrosc. Ra., 110, 533-572, 2009.
Scarnato, B., Staehelin, J., Peter, T., Groebner, J., and Stuebi, R.: Temperature and slant path effects in Dobson and Brewer total ozone measurements, J. Geophys. Res., 114, D24303, doi:10.1029/2009JD012349, 2009.

Voigt, S., Orphal, J., Bogumil, K., and Burrows, J. P.: The temperature dependence (203-293 K) of the absorption cross sections of $\mathrm{O}_{3}$ in the $230-850 \mathrm{~nm}$ region measured by Fourier-Transform spectroscopy, J. Photoch. Photobio. A., 143, 1-9, 2001. 